\title{
The Design of Online Shopping Platform Support System Based on
}

\author{
Web3.0 \\ Tongdi Xiao*,Zhenghua Hu, Libing He \\ Institute of Economics and Management, Nanjing University of Aeronautics and Astronautics, \\ Nanjing 210016, China \\ Email:635641360@qq.com
}

\section{Email:aad998@nuaa.edu.cn}

\begin{abstract}
The business volume of online shopping which is based on the internet will have an inevitable sustained growth, and it can hardly satisfy customers' shopping experience only by the online shopping platform that is form by 2D graphic and text messages. By hold some characteristics of Web3.0 time, this article set the future online shopping platform system, which can visualize the product. The system use Distributed Artificial Intelligence to be its main body frame, mix some modern technology such as Big Data, Web3D and so on, improve customers' shopping experience by visualize the $3 \mathrm{D}$ presentation of products to them through the platform.
\end{abstract}

Keywords: Web3.0; online shopping platform; Big Data; Web 3D technology

\section{INTRODUCTION}

McKinsey Global Institute forecast: By 2020, the size of China's online shopping will reach 2,500 billion to 2,000 billion. However, due to technical and application level, the current online shopping is still in its infancy. Online shopping platform in the products display mainly based on the two-dimensional image and text information, which showed weak commodity performance, platform-independent guide, user's poor interaction, user's weak shopping experience and other shortcomings. Thus, the online shopping market's huge potential and the low user experience by its platform inevitably spawned a study on the future of online shopping platform support system ${ }^{[1]}$.

\section{WEB3.0 FEATURES OF THE TIMES}

Web3.0 is an era of intelligent life which based on networking, big data and cloud computing, it uses a more concise manner to provide customers with personalized information and data, which will be a key step from technical innovation to user concept innovation ${ }^{[2][3]}$.

\section{1 era of big data, structured web content}

The rapid development of networks promote the data and the scale of human society at an unprecedented rate, the era of big data is around the corner, and network data began to change from simple processed to be a basic resource. In the Big Data era, for a huge web content, you need a separate label affixed for the content of each page, index content on the network to achieve the overall structured content, which allows for effective information retrieval.

\subsection{Virtual reality era, three-dimensional network virtualization}

Virtual reality forming a three-dimensional virtual world, provide users with visual, auditory and tactile senses, allowing users to a timely, seamless connection with the three-dimensional space, as immersive. Web3D technology is based on the Internet and virtual reality technology, it based on 
the Internet and software technology, establishing desktop three-dimensional virtual reality world, allowing users to be more clear and intuitive understanding the object of interest [4] [5].

\subsection{Age of Experience Economy, user-driven future}

Web3.0 is a new era of commercial civilization under the "customer first" thinking guidance. Based on the different economic value, economic times can be divided into four stages: cargo, goods, services, experience. The experience stage means that companies will put economic needs of users in the first row, use goods and services as a carrier for the user to create feel which is worth of memories. Users throughout the entire network make the individual needs to the company through various channels, and for enterprises, to understand the market, innovative products, and strengthen the user experience is the most important work.

\subsection{Interconnection of all things, diversity and universality of the terminal device}

Web3.0 is an era to achieve "all things Internet", to meet the user's transformation needs to the network social. At present, China's networking applications has begun to take shape, social-oriented networking has transformed platformization, mainly in the growing of compatibility of the network, terminal equipment, embedded technology and application programs The popularity of faster 4G network services will lead to the rapid release of information, the information more timely, economic life will not be affected by the impact of time and space.

\section{THE OVERALL DESIGN OF ONLINE SHOPPING PLATFORM BASED ON WEB3.0.}

\subsection{Overall concept}

Based on network environment of Web 3.0,this article integrate the Big Data, Web3D, platform ERP system, logistics and distribution and monitoring system, design a future online shopping platform support system. The platform support system is embodied in "E\&3S ", E is short for experience, it is a design of the platform's overall presentation system based on Web 3D technology, which means improving user's experience by the comprehensive three-dimensional virtual online shopping platform, dynamic display and user interaction, "3S(streams)" is the design for information flow, capital flow and logistics systems, forming a Complete information flow loop system by the use of Big Data and System information integration and feedback mechanism, providing decision support for self-learning and improvement of the online shopping platform.

\subsection{Design Principles}

Through spilt the online shopping platform into "E\&3S", the platform support system use Web 3.0 to be its design background, the design process following the principles of "user-centric", strong performance, forward-looking and guidance.

\subsection{System Components}

System design the framework by the distributed artificial intelligence (abbreviated Agent), and treat science and technology as the main body of the system, which is most typical of the Web 3.0 era. Agent module has a self-learning and adaptive capacity, therefore, each subsystem of platform can be run independently in the network environment, to ensure efficient information input and output between subsystems. Online shopping platform support system comprising 10 kinds of Agent subsystem: system management, system evaluation, information integration and feedback, large data searching from both internal and external, and information integration, virtual three-dimensional, virtual information integration, product dynamic display, interactive, logistics monitoring. Each subsystem has different functions, through their collaborative work, the system could have the feature like data searching, virtual product display and interaction platform management. The whole system runs on the Internet. Specific system configuration shown in Figure 1. 


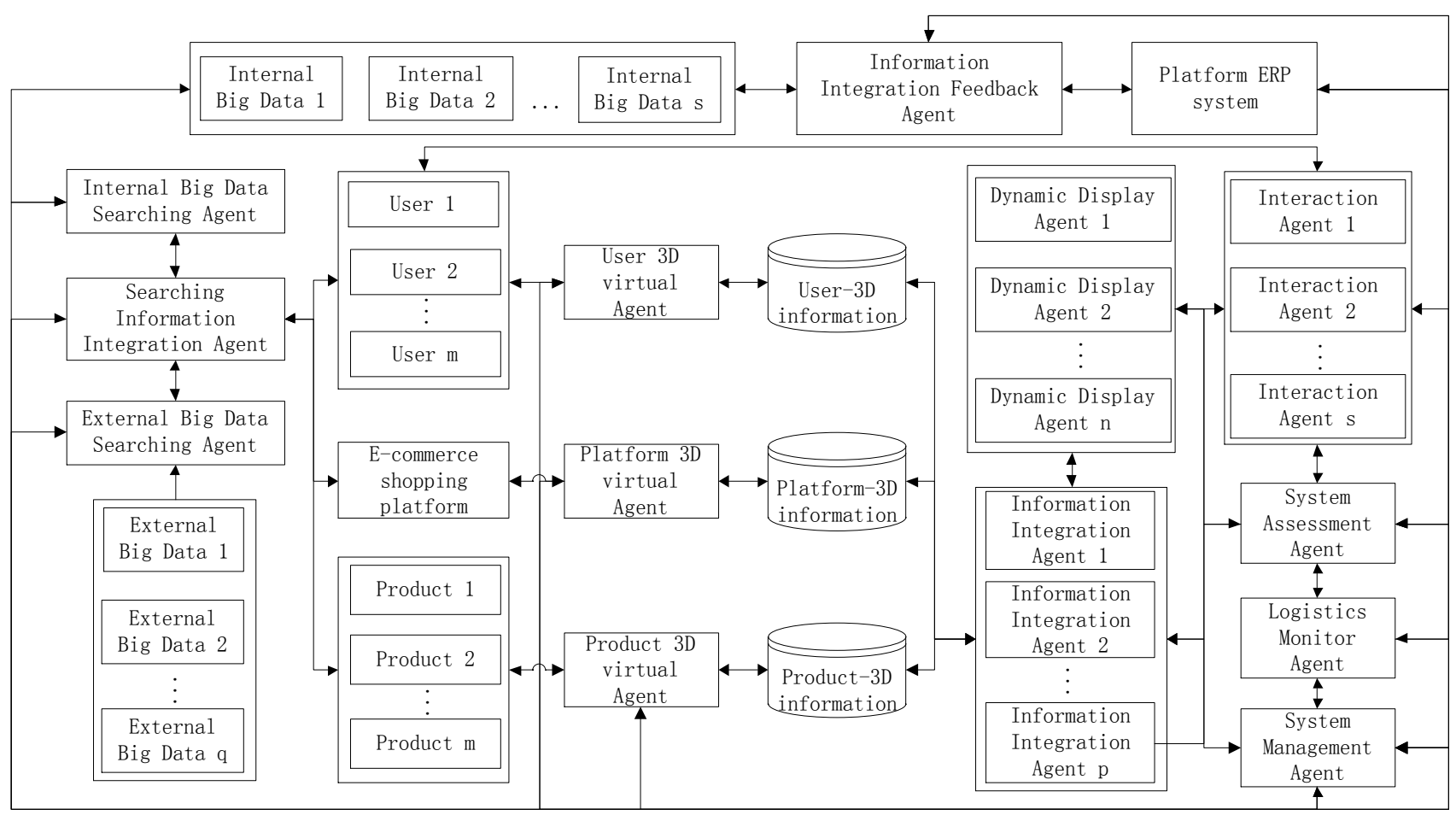

Figure 1 The design of online shopping platform based on Web3.0.

\section{THE FUNCTION DESIGN OF THE SHOPPING ONLINE PLATFORM BASED ON WEB 3.0}

\subsection{The function of System Management of Agent subsystem}

The subsystem is designed to set and maintain the entire online shopping platform support system, besides that, it is also responsible for the connection with other Agent. After enabling platform support system, the Agent subsystem of management needs to set the system initialization and related operating parameters; during normal operation of platform support system, the other subsystems will operate according to their respective objectives and resources setting. At this time, the System management is for establishing a link between subsystems to achieve work together to accomplish the overall goal of Platform Support System.

According to each subsystem's registration information, the establishment of their unique identity, mailing address, type and function information, forming a basis for the exchange of information and transfer, by using the intermediate view technical to exchange data between databases and mail.

\subsection{The function of System Assessment of Agent subsystem}

The subsystem of Evaluation is designed to assess the working ability and the efficiency of all the Agent in the platform, then transmit the assess results to the corresponding Agent, prompting them 
to self-learn and adapt to changing internet environment. It evaluate each Agent in their reliability, validity, reaction speed, adaptive and learning ability in the work process, under different circumstances, the evaluating content will be different. Assessment Agent itself has an adaptive and self-learning ability, it will adjust the content and criteria of evaluation as the environment change. Meanwhile, as part of the large internal data, the results of the assessment also provide decision support for the system re-design.

\subsection{The function of Information Integration Feedback of Agent subsystem}

The subsystem of Information Feedback is a connection of all Agents and large data system. Its key features include two aspects, one is to integrate the information both from each Agent and ERP system; the second is to provide data support for the products updating by feedback in some classification index to the internal large data systems.

\subsection{The function of Big Data Searching and Information Integration of Agent subsystem}

This part include the subsystem for big data searching from both internal and external, and the subsystem for information integration. The former identifying potential needs of users by achieve classified aggregation and searching analysis of internal and external data, looking for the most efficient match between the platform and users by combing the users' potential need and the analysis data. The latter is to integrate the searching data in accordance with pre-designed index, providing the necessary data support for business platform to improve their users' experience. As the key technology of Great data analytics, cloud computing can quickly process huge amounts of data, its key contents include: file system technology, distributed database systems, database indexing system and big data analysis techniques.

\subsection{The function of Three-dimensional Virtual of Agent subsystem}

This Agent include Platform Virtual, Product Virtual and User Virtual. Platform Virtual Agent is designed to build a virtual building style shopping platform to provide users with virtual reality mall shopping experience; Agent Products Virtual aimed at virtual the business products in three-dimensional, to provide users with the products' look, function, structure and other comprehensive information display, and provide the product trial; User Virtual Agent make it possible for user roaming in the virtual construction platform users by virtual characters. The Three-dimensional Virtual Agent finally fused platform, products and users together, virtualizing users to try virtual product in a virtual mall, allowing users to feel the same experience as offline shopping. Depending on the different objects, different technologies such as Cult3D and QTVR can be used to complete the three-dimensional modeling.

\subsection{The function of Information Integration of Agent subsystem}

Information Integration Agent is designed to storage the integrated information of user, platform and product orderly in the right area, and transferred it to Dynamic Display Agent by the use of an intermediate view technology. This Agent subsystem has two functions, one is to achieve integration of information form user, three-dimensional virtual platform and product, the second is to transfer different information to different areas, based on the storage partition the platform use for separating businesses and the users.

\subsection{The function of Dynamic Display of Agent subsystem}

This subsystem intended to convert integrated three-dimensional information into an image than user can see. Its function in two ways: First, to ensure users' true feelings of virtual scene by achieving seamless connection among the user, products and three-dimensional model of the platform; the second is based on the internet: a roaming of three-dimensional scene on the online shopping platform, allowing users to freely navigate between different three-dimensional online shop and three-dimensional products by achieving three-dimensional scene switch in various forms. 


\subsection{The function of Interaction of Agent subsystem}

The interaction Agent is an interactive interface of the online shopping platform support system, it is a connection between the user and the online shopping platform. This part has three functions, namely the provision of advertising support, so that the product or service on the online shop and the platform can obtain comprehensive presentation and display; the second is to provide users with comprehensive demand product; the third is to provide the user with guidance of the shopping intelligent operation, enhance the user's shopping experience. Users can interact with the online shopping platform through various types of mobile terminals.

\subsection{The function of Logistics Monitor of Agent subsystem}

Logistics Monitoring Agent is designed to provide information services of logistics and distribution process for clients of online shopping platform. It accomplish two main functions, one is to integrate the distribution information of express, for the clients getting the latest information about the express delivery. Second, grasp information of all delivery routes in the platform and the feedback from the customers, facilitate alliances courier companies to optimize delivery routes and improve service quality. It can accurately obtain the specific location of the express in the distribution system by using the technology of RF, GPS (or BDS), vehicle tracking and tag identification.

\section{CONCLUSION AND OUTLOOK}

As the rapid development of the Internet, Web2.0 era will inevitably become history, in the Web3.0 era, it will be very important how to accurately grasp the network development context. Taking Agent as the main framework for the design of the "E\&3S " online shopping platform support system, this system integrate the big data, cloud computing, Web3D technologies, platforms ERP systems and logistics monitoring system to ensure the information acquisition of the whole platform and sharing of resources, and also meet the customers' demand for user experience and diversified. Although the efficient integration of all types of modern technology in the online shopping platform system design process is still to be verified, three-dimensional virtual online shopping platform is bound to be the development trend in the future.

\section{References}

[1] WenXiao Guo. The future development trend of e-commerce [J]. SMEs management and technology, 2013, (11):159-160.

[2] Yan Cai. Construction of University Libraries Self-learning platform in Web3.0 [J]. Modern Information, 2014, 34(3):82-86.

[3] Qipin Lin. The design and visualization of the network 3D display system based on Web3D. [J].Information Science, 2011，29(5):692-695.

[4] Weiqiao Zhu. Library Functions Design Based on Big Data. [J]. Modern Information, 2014, 34 (8) :83-86.

[5] Xiaofeng Meng. Management of Big Data: Concept 、Technology and Challenge [J]. Computer Research and Development, 2013, (1):149-167.

[6] Qiming Cai. The Design of forecast support system based on Internet/Intranet [J]. Information Science, 2004, (7):857-861. 
[7] Hui Fang, Jie Zhang, Murat Sensoy, Nadia Magnenat-Thalmann. Reputation mechanism for e-commerce in virtual reality environments $[\mathrm{J}]$. Electronic Commerce Research and Applications, 2014(8):1-14.

[8] Xianmei Jiang. Research and Virtualization of the e-commence display platform based on Virtual reality technology [D]. Jiangxi Science and Technology Normal University, 2010. 